\title{
"KMS-Fit": a case-based exploration of task/technology fit in an applied knowledge management context
}

\author{
Jason M. Turner* \\ Graduate School of Engineering and Management (AFIT/ENV), Air \\ Force Institute of Technology, 2950 Hobson Way, Wright-Patterson \\ AFB, OH 45433-7765, USA \\ E-mail: jason.turner@us.af.mil \& jundlt@yahoo.com \\ *Corresponding author

\section{David P. Biros} \\ 415 Business Building, Spears School of Business, Oklahoma State \\ University, Stillwater, OK 74078, USA \\ E-mail: david.biros@okstate.edu

\section{Michael W. Moseley} \\ National Air and Space Intelligence Center (NASIC/SC), 4180 Watson \\ Way, Wright-Patterson AFB, OH 45433, USA \\ E-mail: michael.moseley@wpafb.af.mil
}

\begin{abstract}
The notion of Task/Technology Fit (TTF) posits that as the degree of overlap increases between the task domain, and the ways in which the capabilities of an information system (IS) are suited to activities within that domain, performance gains experienced via use of the IS should also increase. This research proposes an expanded TTF model that is applicable to the context of Knowledge Management (KM) and Knowledge Management Systems (KMS). In particular, additional individual, technological, and social factors and interrelationships between these factors could provide greater explanatory power of IS user behaviors, perceptions, and outcomes within the realm of knowledge work.

A mixed-method field study approach was employed at a large government organization, currently in the process of developing and fielding a new KMS to support knowledge-intensive work, to investigate the underlying factors and relationships described within an expanded "KMS Fit" model. Results suggest that the foundational mechanisms described by the TTF model may in fact change within KM contexts. In particular, the inherently social characteristics of knowledge-based work were found to play a very important role in determining the degree of fit relative to a KMS. Moreover, the social ecology within the organization was found to have significant impact on KMS Fit. Results of this research further reinforce the notion that KMS may be a unique subset of IS and that traditional IS models (such as TTF) should be updated or tailored to reflect the social nature of knowledge-based work and knowledge management.
\end{abstract}

Keywords: Task-Technology Fit, KMS-Fit, Knowledge Management Systems, Social Ecology 


\begin{abstract}
Biographical notes: Dr. Jason Turner is a lieutenant colonel in the United States Air Force and is currently director of the Air Force Institute of Technology's Graduate Information Resource Management Program. $\mathrm{He}$ earned his $\mathrm{PhD}$ in information science from the University of Texas at Austin. His research interests include psychological, social, and organizational impacts and uses of information and information technology.

Dr. David Biros is an Assistant Professor of Management Science and Information Systems at Oklahoma State University. His research interests include deception detection and system trust. He has been published MIS Quarterly, Decision Support Systems, Group Decision and Negotiation, MISQ Executive, and the Journal of Digital Forensics Security and Law.

Mr. Michael Moseley is a captain in the United States Air Force and recent graduate of the Air Force Institute of Technology's Masters Program in Information Resource Management. He presently serves as Executive Officer to the Director of Communications and Information at the National Air and Space Intelligence Center.
\end{abstract}

\title{
1. Introduction
}

\subsection{Background}

Contemporary definitions of knowledge tend to center on the mind of the knower: justified, personal beliefs that increase one's ability to take decisive action (Alavi \& Leidner, 1999; Nonaka, 1994) — often a complex amalgam of "framed experience, values, contextual information, and expert insight that provides a framework for evaluating and incorporating new experiences and information" (Davenport \& Prusak, 2000, p. 5). When managed effectively, knowledge can provide increased efficiencies and competitive advantage (Argote \& Ingram, 2000; Davenport \& Prusak; Osterloh, 2000). The practice of Knowledge Management (KM) identifies and mobilizes knowledge resources, turning them into value-creating activities (von Krogh, 1998). KM activities tend to fall along lines of four basic processes: creation, storage/retrieval, transfer, and application (Alavi \& Leidner, 2001). As a discipline, KM has been rooted in action, requiring knowledge be used and applied before it can ultimately impact an organization (Jennex, 2008).

Such impact can be profound and knowledge management is now regarded as one of the cornerstones of business success-according to a report by INPUT, the US Government spending on knowledge management solutions is projected to reach $\$ 1.3$ billion by fiscal year 2010; a 35 percent increase over then existing KM expenditures (INPUT, 2005). Despite this significant investment, however, there is no guarantee that knowledge management projects will attain their objectives. Storey and Barnett (2000) report that the majority knowledge management projects fail to have any real impact.

Information technology (IT) has also been applied to knowledge work and knowledge projects in an effort to increase the efficiency and effectiveness of organizational processes. Knowledge Management Systems (KMS) are "IT-based systems developed to support and enhance the organizational processes of knowledge creation, storage/retrieval, transfer, and application" (Alavi \& Leidner, 2001, p. 114). Given the importance and potential impact of knowledge within organizations, the proper design, acquisition, and application of KMS have become major thrust areas for managing and leveraging organizational knowledge (Huber, 2001). In 2007, US 
companies alone spent an estimated $\$ 73$ billion on knowledge management software (McGreevy, 2007).

However, much like investments in other KM initiatives, “...organizational investments in computer-based tools to support planning, decision-making, and communication processes are inherently risky" (Davis, Bagozzi, \& Warshaw, 1989, p. 982). Those rushing to procure KMS may do so under the pretense that knowledge moves without friction or motivating forces, or that the organization's employees are simply vessels of knowledge waiting to spill experiences and insight onto others "...with no concern for what they may gain or lose by doing so" (Davenport \& Prusak, 2000, p. 26). Organizations should therefore strive to understand the complex nature of the environments in which KMS are deployed lest they find their investments failing to produce optimal results.

\subsection{Research focus}

Chua and Lam (2005) recently described several cases detailing but a few of the many reasons why KM programs or systems might fail. Another potential source of such failure may involve a lack of "fit" between the KMS and the organization implementing the technology. Task/Technology Fit (TTF) (Dishaw \& Strong, 1999; Goodhue, 1998; Goodhue \& Thompson, 1995; Mathieson \& Keil, 1998) describes a framework by which some of the factors and risks associated with applying IT (or KMS) to organizational processes and activities can be identified and explored. TTF explicitly posits the notion of Fit, an overlap or match between the capabilities of an information system and the task(s) for which it was designed - the greater the degree of fit, the more likely a rational individual would (or should) employ the system and the more likely employment would positively impact performance. However, the context in which organizational activities are executed may also impact knowledge processes. The success of a KMS may therefore be dependent upon fit as well as the context or environment in which the KMS is used.

Through better understanding of the contextual factors that could impact KMS employment, those who develop, acquire, or deploy KM applications and systems might be able to increase the likelihood of success (Dishaw \& Strong, 1999). This research attempts to shed light onto the nature of TTF cast against the backdrop of KM, and explore the relevant features and dynamics of the context of KMS use that may ultimately impact system success. The following questions frame the remainder of the study:

Do established notions and mechanisms of TTF differ in the context of KM and KMS? If so, how are the differences manifested?

The following sections examine the viability of a KMS-oriented TTF model within the context of a large US government organization. A review of foundational perspectives on knowledge and KM is first presented. An expanded TTF or "KMS-Fit" model is then conceptualized and introduced. The model is then evaluated using field data collected from knowledge workers. Finally, results from the evaluations and recommendations are offered to aid those developing or employing KMS as viable tools for managing organizational knowledge.

\section{Literature Review}

\subsection{Perspectives on knowledge and knowledge management}

Cognitive perspectives of knowledge often describe mental representations of the world; the key task of any cognitive system (such as the brain) is to model those representations as accurately as possible such that "two cognitive systems should achieve the same 
representation of the same object or event" (von Krogh, 1998, p. 134). Such unanimity of representation and interpretation implies that knowledge is explicit and can be encoded and articulated by formal, systematic, or symbolic languages (Alavi \& Leidner, 2001; Nonaka, 1994). A commensurate knowledge-oriented strategy therefore "...focuses on codification and storage facilities, where knowledge is stored in the form of information in databases, documents in document management systems, and so forth, where it can be accessed by employees" (Baloh, 2007, p. 28).

As Polanyi (1962) noted however, "There are things that we know but cannot tell" (p. 601). The constructionist perspective conceives of knowledge as an act of construction based on factors and inputs from others within the environment that are then placed into context. The notion of explicit knowledge is complemented by the tacit - that which is embedded in the brain, highly personal, and not easily expressed (Grover and Davenport, 2001; von Krogh, 1998). Nonaka (1994) described tacit knowledge as "...deeply rooted in action, commitment, and involvement in a specific context" (p. 16). Tacit knowledge is often social in its origin-people with questions connect, meet, and work with others who have the answers so that tacit (and explicit) knowledge is transferred (Baloh, 2007). Support for tacit knowledge transfer can include collaborative tools to channel expertise, facilitate conversation, and help locate knowledge holders (Baloh, pp. 28-29).

Neither perspective alone provides a complete picture. An appropriate or effective KM strategy is ultimately dependent on the business context and processes, and different knowledge needs (tacit or explicit) may call for different approaches to KM (Baloh, 2007). KM per se helps determine what knowledge is valuable and where knowledge should be distributed or applied to improve decision-making and the ability take effective action (Jennex, 2008; Jennex, Smolnik, \& Croasdell, 2007). Objectives of KM are: "to make the enterprise act as intelligently as possible to secure its viability and overall success...to otherwise realize the best value of its knowledge assets" (Wigg, 1997, p. 1).

Jennex and Olfman (2004a) identified 12 key KM success factors distilled from 14 different studies that evaluated a total of $78 \mathrm{KM}$ initiatives. A recurring theme among those factors was the social or contextual aspect of KM; for example, a culture that supports learning and knowledge sharing. These findings suggest that KM can be a largely social discipline, and that "Success with 'managing knowledge' will therefore ultimately depend on a manager's sensitivity to people issues" (von Krogh, 1998, p. 134). Gupta and Govindarajan (2000) further maintain that, "Building an effective social ecology... is a crucial requirement for effective knowledge management” (p. 71).

\subsection{The social ecology}

Social ecology refers to the social systems (culture, structure, information systems, reward systems, processes, people, and leadership) in which people operate to accomplish their jobs - all of those social elements that may impact individual behaviors (Gupta \& Govindarajan, 2000). Ecology suggests that this system is not a set of random, disparate elements, but an interactive set of factors that continuously affect each other. Four elements: knowledge markets, cognitive barriers, knowledge networks, and organizational culture, stand out amongst the universe of potentially relevant features of a social ecology based upon their prevalence in KM-related literature and applicability to the underlying models of KMS success explored later in this analysis.

Knowledge can enable good decision-making, but it must be transferred from the point of origin to the point of decision. Organizations must therefore understand the forces that cause knowledge to move before implementing initiatives attempting to make 
knowledge move (Davenport \& Prusak, 2000). Such movement has been compared to market-like forces where knowledge workers exchange units of knowledge-based currency for present or future value (Davenport \& Prusak). Three forms of currency serve to motivate knowledge flow within knowledge markets: reciprocity, repute, and altruism.

Reciprocity is an expectation of an exchange such that, "A knowledge seller will spend the time and effort needed to share knowledge effectively if he expects the buyers to be willing sellers when he is in the market for their knowledge" (Davenport \& Prusak, 2000 , p. 32). Repute is a perception such that others "...know [a knowledge seller] as a knowledgeable person with valuable expertise that he is willing to share with others in the company" (Davenport \& Prusak, p. 32). Repute is used when employees seeking a certain expertise single out the more reputable sellers in an effort to increase the "quality" of the purchased knowledge. Altruism is akin to intrinsic motivation: knowledge shared for the sake of satisfaction without need of reward. "Many knowledge sharers are motivated in part by a love of their subject and to some degree by altruism, whether "for the good of the firm' or based upon a natural impulse to help others" (Davenport \& Prusak, p. 33). When employees weigh the decision to share their knowledge, they compare the value of their knowledge with the perceived value of one or more of these forms of currency which serve as mediums of exchange for knowledge transactions.

Cognitive barriers are issues that prevent a knowledge seller and buyer from arriving at a shared understanding; they affect how and to what extent individuals share knowledge with others. Cognitive barriers are most often encountered when dealing with tacit knowledge (Huber, 2001), often because communicating such knowledge typically requires unconventional language techniques (analogies and metaphors) to convey meaning to those not already knowledgeable in the subject matter (von Krogh, 1998). Thus, the foundation for shared meaning (much less knowledge) is a shared languageone that is often developed only through repeated knowledge transactions and slowly breaking down former cognitive barriers. Tacit knowledge also tends to be "sticky" (highly entwined with cognitive processes), making such knowledge especially difficult to articulate and therefore exacerbating or creating additional cognitive barriers (Huber).

Research indicates that the presence and strength of knowledge networks - a community of individuals brought together by a common interest-can impact the success of KM initiatives (Davenport \& Prusak, 2000). "When networks of this kind share enough knowledge in common to be able to communicate and collaborate effectively, their ongoing conversation often generates new knowledge within firms" (Davenport \& Prusak, p. 66). For example, when knowledge workers use conversations to trade "highly informative war stories," they are in fact managing knowledge (Davenport \& Prusak, p. 45). In a strong knowledge network, this process occurs many times over, allowing for knowledge to be applied over a broad set of tasks. The strength of such networks comes from the communication between members; the greater the degree of communication within the network, the greater the impact on the flow of knowledge throughout the organization (Brown \& Duguid, 1991).

Finally, organizational culture is "...the set of values, beliefs, norms, and expectations that are widely held in an organization" (Huber, 2001, p. 76). Organizational culture can manifest itself through stories and habits (von Krogh, 1998). Stories can highlight failed attempts to implement a technology, pursue a new market opportunity, or develop a new product. Habits are routines that are difficult or even impossible to turn and they can hold an organization back from reaching maximum potential (von Krogh).

Formal procedures can also define organizational culture (von Krogh, 1998). Formal procedures represent embedded experiences and the successful solutions to complex tasks 
that are codified and adopted as policy. A KM initiative hinging on restrictive formal procedures runs the risk of interfering with the natural flow of information or inclination of organizational members to enter into knowledge transactions. In fact, too much managerial oversight or regulation can ultimately reduce the effectiveness of existing knowledge networks (Davenport \& Prusak, 2000).

An organization's paradigm, or lens through which it views the world, strongly influences its culture (von Krogh, 1998). Paradigms may be expressed in terms of strategic intent, vision or mission statements, strategies, and core values (von Krogh, p. 136). An organization's paradigm does not develop overnight, but slowly over time, shaped by the experiences of the organization. Like habits, an organization's paradigm can be extremely hard to change-knowledge processes can be stifled by the ruling paradigm if it gives rise to an environment not conducive to knowledge-based exchanges or communication within and between knowledge networks (Davenport \& Prusak, 2000). Thus, the influence of ruling paradigms must be considered when evaluating the suitability of a particular KMS to a particular organizational setting.

\subsection{Proposed model of "KMS Fit"}

KMS are tools designed to manage organizational knowledge, to include the processes of knowledge creation, storage/retrieval, transfer, and application (Jennex \& Olfman, 2004a). If the social ecology is a vital consideration for a successful KM strategy, a successful KMS must logically integrate into the established social ecology as well. But how do we know if a KMS is doing what it should as successfully as possible? The traditional and revised IS Success Models (DeLone \& McLean, 1992 \& 2002) and more specific KMS Success Model (Jennex \& Olfman, 2004b) both suggest that system (KMS or otherwise) success is a multidimensional set of interrelated constructs, assessments, and perceptions based on system and service characteristics, input (knowledge or information) quality, and user-related behaviors.

Of particular importance to this investigation is Intent to Use-conceded as a potential substitute for Use in the revised IS Success Model (DeLone \& McLean, 2002) - intent was later identified as a core construct in the KMS Success Model (Jennex \& Olfman, 2004b). Jennex (2005) proposes that KMS success is not based upon amount of usage but user intent because, "End users stated that it was knowledge used infrequently that was knowledge with the greatest value and impact. This implies that the KMS with the greatest impact is the KMS that may not be used all that frequently" (p. 7). Similarly, Jennex (2008) observed that, “...it was not how often [the interviewees] used the KMS but rather it was the one time that they absolutely had to find knowledge or found unexpected knowledge that proved the worth of the KMS" (p. 58). Such observations that Intent to Use, versus Use, may be appropriate for assessing systems success (DeLone \& McLean, 2002; Seddon \& Kiew, 1996) provide a foundational argument for KMS as a unique research context, one requiring models tailored for more effective measurement and understanding (Jennex \& Olfman, 2004b, Jennex, 2005 \& 2008).

Just as the IS Success Model was adapted for KMS contexts, the same may be appropriate for TTF-a model also having roots in traditional IS literature. TTF (Figure 1 below) is defined as the degree to which characteristics of a technology fit the task it was designed to support (Goodhue \& Thompson, 1995). TTF implies that the value of an IS is dependent upon how effectively and efficiently the system helps its users complete a task or collection of tasks (Goodhue, 1998; Mathieson \& Keil, 1998) - the higher the degree of fit, the better performance is likely to be (Goodhue \& Thompson). 


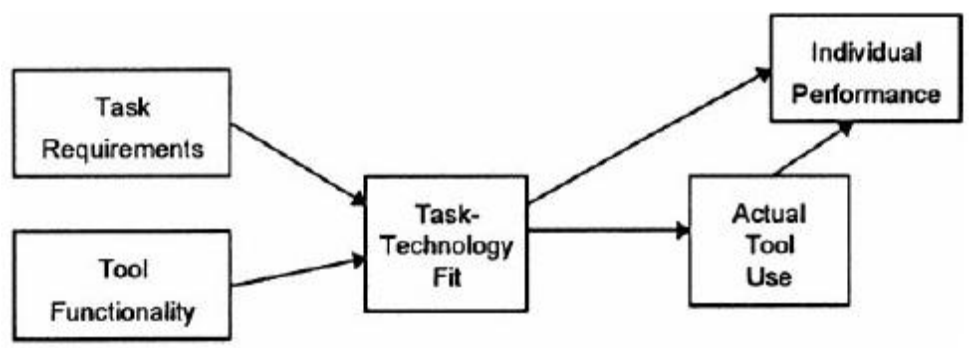

Figure 1. TTF Model (Dishaw \& Strong, 1999)

Task Requirements are those activities or functions required to turn input into output (Goodhue \& Thompson, 1995); Baloh (2007) suggests this construct captures the nature of the work and the kind of knowledge involved. Baloh proposes two specific task domains within the context of KM: focused (relying on functional knowledge of a specific area) and broad (relying upon general knowledge from a variety of processes within an organization). Tool Functionality describes the capabilities and design features of a tool including hardware, software, and data characteristics as well as the services designed to support them (Goodhue \& Thompson). Tool Use is simply the act of employing the technology to complete user tasks (Goodhue \& Thompson). Individual Performance refers to "...the accomplishment of a portfolio of tasks by an individual" (Goodhue \& Thompson, p. 218), although, like Use, performance could be measured many ways including production efficiency, completion time, or decision quality (DeLone \& McLean, 1992) - the most appropriate measure tends to depend upon the nature of the question being asked and the business context.

A critical component of that context within the realm of KM is the social ecology and its constituent elements. If the TTF framework is to be extended to the specific research context of KMS, the explanatory power of the model may also be improved by incorporating social factors that comprise the business context. An examination of every possible permutation of TTF within the social ecology is beyond the scope of the current investigation. Instead, a tailored model of KMS Fit is proposed that accounts for some of the more likely impacts of the social ecology on the underlying mechanisms of TTF.

For example, Davenport and Prusak (2000) maintain that the dynamics of knowledge markets and market forces are critical when developing or implementing KM initiatives such as a KMS. Ultimately, knowledge market forces describe the patterns and mechanisms of influence to participate in KM initiatives - theoretically, such influence should likewise impact intention to use a KMS. Knowledge networks were described as social constructions that help move knowledge within an organization; the efficacy of that network, or the ability of a KMS to connect members within a network, may also exert influence on one's intention to employ a particular KMS.

Cognitive barriers impact the ability to reach shared understanding. These barriers may hamper identification and definition of knowledge task requirements, especially if the subject matter is not entirely familiar, the task is especially complex, or resides mostly in the minds of other employees. Similarly, cognitive barriers may stand in the way of KMS use per se, or limit the ability to properly conceptualize how various KMS design features and/or use can enhance performance. Finally, organizational culture can exert a powerful influence on the actions and perceptions of organizational members. Procedures, habits, and paradigms undoubtedly exert strong influence over the conceptualization of the knowledge task itself, as well as the attitudes and perceptions about a particular KMS, or the use of KMS in general within the organization. 
Figure 2 illustrates a proposed model of KMS Fit - a starting point for the current investigation and a framework for consideration and analysis of the obtained results. Commensurate with prior studies, Use is replaced with Intent to Use. Furthermore, Performance is defined as "the degree to which an individual is able to accomplish a task or number of tasks" - the implication is that KMS use per se has occurred, is occurring, or was not necessary. Consequently, the direct relationship between TTF and Performance was removed. The following section will describe the methods by which the underlying factors and interrelationships of the proposed KMS Fit model were analyzed.

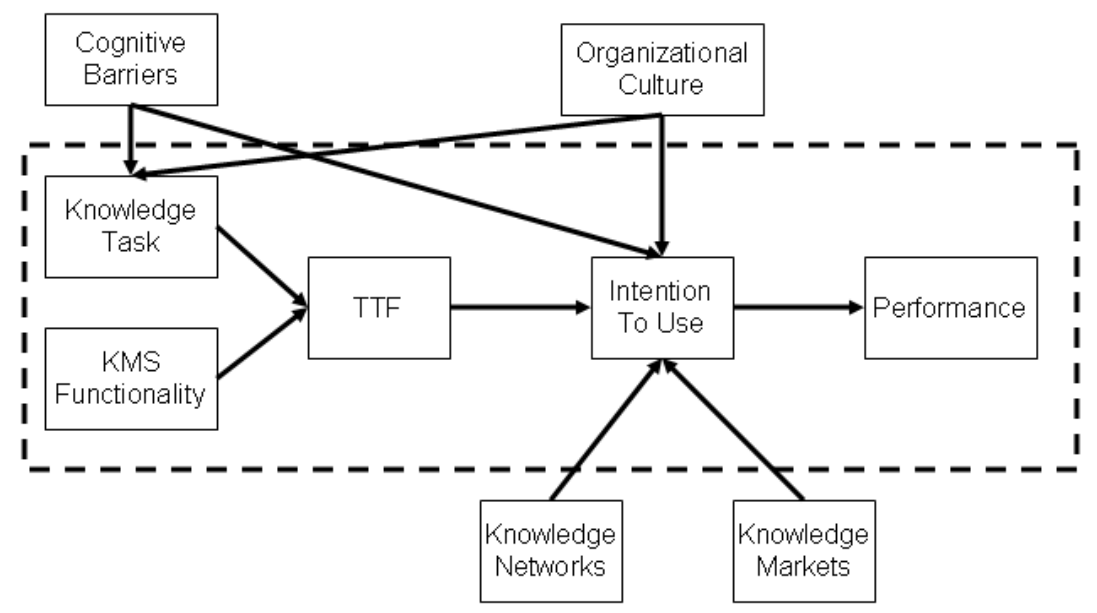

Figure 2. Proposed model of KMS Fit (underlying TTF model highlighted)

\section{Methodology}

Appreciating the complexities of TTF and the social ecology in a KMS context requires immersion, deep understanding, and the ability to identify and analyze the many nuances at work within the social context surrounding KMS use. Such requirements are indicative of Yin's (2003) questions of "what" - what role, if any, do the social aspects of KM play in the fitness of KMS to the knowledge tasks for which they are designed. Consequently, a field study-like approach was selected and designed to accommodate a series of focused, inter-related, and semi-structured analyses to explore KMS Fit from the many perspectives of those with first-hand experience of the issues under investigation.

\subsection{Research context}

The Defense Ammunition Center (DAC), a large Department of Defense organization currently developing and fielding a new KMS, was selected as the research site. The DAC provides ammunition training, support for explosives safety, demilitarization research and development, and logistics engineering support including supply, maintenance, and transportation. The DAC also manages two Army career programs for ammunition expertise providing 58 training courses to personnel across many disciplines. Such diversity made the DAC an ideal site for study - it creates new knowledge through the engineering directorates, transfers knowledge through the training directorates, and executes that knowledge both internally and for external customers. 
An aging and retiring workforce is quickly draining the DAC of its experience, training, and know-how. The DAC is therefore developing and fielding the Expertise Transfer System (ETS) to capture knowledge from employees and present it in a shareable, searchable, and flexible form. Specifically, individuals are interviewed and their transcripts mined via search algorithms to construct collections of causal statements which are packaged into graphical or textual representations of the knowledge content. These representations are incorporated into the ETS and posted on the DAC's enterprise network via the ETS portal.

\subsection{Participants}

Participants consisted of DAC employees who attended an instructor training course at Oklahoma State University in early 2008. Participation was voluntary though the study was sanctioned by the DAC leadership and results were used to aid in the development of the ETS. Of the eleven students in the course, seven volunteered to be interviewed for a response rate of 63 percent. Four of the respondents were male and three were female. Average age was 46 years (standard deviation 10 years); average work experience at the DAC was 3 years (standard deviation 1.5 years); average time in the ammunition career field was approximately 8 years (standard deviation 2 years).

\subsection{Procedures and data collection}

Using the proposed KMS Fit model as a foundation, a semi-structured interview protocol was developed and administered based on the Interactive Qualitative Analysis (IQA) methodology (Northcutt \& McCoy, 2004). IQA seeks to capture "lived realities" of individuals and their experiences, directly engaging participants in depicting their experiences which ultimately describe a collective understanding of a given phenomenon. First, respondents were queried concerning their experiences and understanding of the constructs in the KMS Fit model as they pertained to knowledge work in the DAC and the ETS, as well as the DAC's culture, knowledge markets, knowledge networks, and their perceptions or experiences of cognitive barriers. Second, respondents described their perceptions and experiences of how each construct influenced, impacted, or was related to each other construct (if at all). For example, participants were asked if cognitive barriers impacted KMS functionality, if functionality impacted cognitive barriers, or if the two were unrelated. If respondents were ambivalent but perceived a connection, they were asked to describe the relationship that was most prevalent or most salient.

The IQA procedures in this study differed from the traditional IQA methodology in two ways. First, IQA respondents are often asked to help develop and identify the various constructs involved in their understanding of how a perceptual system or process behaves. Here, the constructs were provided - and therefore pre-defined - as part of the research model. Second, all possible combinations of influence or effect between the constructs in the KMS Fit model were not examined. For instance, there is no direct relationship between the task requirements and individual performance in the TTF model. Similarly, participants were not asked how they experienced the impacts of the knowledge task requirements on performance or vice versa - only the relationships mirroring the TTF were examined as threshold check of whether the TTF functioned "as advertised" relative to KMS. However, the impacts of the social ecology factors upon each TTF construct are largely unexplored; therefore, each possible pairing of the social ecology factors with TTF constructs was examined to explore how the social ecology might impact any of the underlying mechanisms of TTF in a KM context.

Tallies were collected across all participants indicating how often one construct was perceived to influence another. For instance, one tally was generated for the number of 
respondents indicating that cognitive barriers impacted KMS functionality, another for KMS functionality impacting cognitive barriers, and another if no relationship was perceived. Individual tallies were then represented by a structural model involving all constructs and relationships in accordance with IQA's data reduction and representation techniques. Results of the analyses were used to develop a perceptual and experiencedbased model, founded upon TTF constructs, to depict how (if at all) various social factors may have impacted each of those constructs, and how well the model itself described the context or mechanisms underlying this particular KMS implementation.

\section{Results and Analyses}

Results follow a format similar to the interviews, focusing first on the constructs in the research model. The interrelationships between constructs will then be examined. Finally, a model derived from participant data will be presented and discussed concerning the nature of TTF in the context of the DAC's KMS implementation and KMS use.

\subsection{Construct analyses}

Interviews were transcribed verbatim and then segmented based on corresponding interview questions. Individual passages were highlighted and iteratively grouped into major themes via open coding until all passages were accounted for. Iterative refinement within each theme yielded unique sub-components. Counts were maintained of the total number of respondents expressing sentiments congruent with each component to provide a sense of the prevalence or minority/majority perspective on that particular subject.

\subsubsection{Knowledge task requirements}

This construct centered on the knowledge processes of creation, storage/retrieval, transfer, and application. Respondents felt that knowledge was created through field experiencethen applying what is learned to other real-world situations. However, many respondents perceived their role as knowledge sharers rather than creators- their most important task was to make knowledge available to others.

Knowledge storage was articulated in terms of technology (digital documents) and in the minds of students. For the latter, rote memorization was de-emphasized in favor of imparting the tools necessary to allow others to think critically and enable problemsolving. Such perceptions arose from the sheer bulk of information associated with ammunitions-related work, and from the fact that munitions knowledge is volatile, requiring continual refresh. Discussions also centered on the DAC's library and extensive database - the library was cited as an indicator of a positive, knowledge-oriented culture.

Knowledge transfer was uniformly articulated in terms of knowledge flow from instructor to student - transfer occurred during classroom instruction, hands-on, and onthe-job training. In a similar vein, knowledge application was perceived as demonstrable performance on exams or briefings to classmates - opportunities to apply knowledge gained to solve presented problems. Almost unanimously, respondents reported that knowledge should enable some sort of action, whether in the classroom or on the job. These results were not surprising given the participants' primary role as instructors.

\subsubsection{KMS functionality}

Aside from seemingly reasonable observations concerning system responsiveness or user-friendliness, respondents indicated that a good KMS should contain validated 
knowledge - especially important given the subject matter (injury or death is possible via mishandling of munitions). Some respondents wanted to see support for a vetting process whereby subject matter experts could review knowledge content. Strong search algorithms and attribution or evidence of authorship were also cited as important to good KMS design, especially in cases where the context from which knowledge was derived was ambiguous or unfamiliar.

\subsubsection{Task/Technology Fit}

Respondents agreed with the formal definition of TTF; the concept itself was intuitive and self-evident. When articulating what might impact the degree of fit between a KMS and a knowledge task, responses were varied. One participant suggested that KMS should be developed by people experienced with the specific task, not just in designing KMS. A large body of information from which to query was also perceived as important to TTF. One respondent also offered that TFF for knowledge work would be improved by a tool that aided in finding or talking to a person when questions arose.

\subsubsection{Intention to use}

Participants provided many examples that described when their intentions to use a system did not translate into actual use. Among the issues discussed were lack of training or expertise with the system itself, mandatory use of a new system that had not proven its worth or reliability, and frustrations based on technical issues such as lack of access or appropriate permissions. Factors that were reported to positively influence intentions to use a system included query response speed and user friendliness of the interface.

\subsubsection{Performance}

Respondents could not imagine accomplishing their jobs without the aid of a computer system - the ETS in particular was cited for improving performance though knowledge transfer (personally, or to others through in-class instruction) and as an aid for knowledge application to new problem sets. However, the promise of the ETS was a double-edged sword. Concerns regarding decreased performance were attributed to the center of gravity the ETS would become. Specifically, as the amount of knowledge in the ETS increased, demands on knowledge owners would also increase through management of the knowledge content and fielding of questions from colleagues seeking clarification. Validating knowledge content was also of concern-reduced performance after "being burned" by faulty information led to reduced trust and ultimately less usage of the ETS.

\subsubsection{Cognitive barriers}

Research suggests cognitive barriers can impede the transfer of knowledge that is especially complex or context-specific (Huber, 2001). Respondents perceived their jobs as fairly complex (average rating of 7.5 on a 1-to-10 scale) and spent a significant amount of time ensuring they had the most current information possible. Such complexity was cited as the primary cognitive barrier in the DAC, arising from the ever-changing stateof-art and inherently dangerous nature of munitions. However, a willingness to share and communicate knowledge to others was uniformly cited as the means by which cognitive barriers associated with this complex knowledge set were overcome; personnel unwilling to share knowledge were singled out for increasing cognitive barriers.

\subsubsection{Knowledge networks}

Respondents were very sensitive to the presence and value of knowledge networks; they routinely used organizational phone books or specific "well-connected" individuals for locating needed expertise (consistent with Jarvenpaa and Majchrzak's (2005) notion of 
ego-centric groups). However, the global nature of the DAC organization hampered communication within the network itself. Respondents often highlighted difficulties associated with leveraging their network contacts due to schedule conflicts or geographic separation.

\subsubsection{Knowledge markets}

Altruism was the most commonly cited knowledge-based currency exchanged within the DAC - employees shared knowledge because "it is the right thing to do." Repute was also deemed important though not for personal reasons or satisfaction, but for the criticality of vetting and verifying the knowledge required for munitions-related work as coming from an authoritative, expert, or otherwise knowledgeable source.

\subsubsection{Organizational culture}

Organizational culture was the most complex and broadly interpreted construct of any in the research model. Discussions indicated a keen awareness and appreciation for what it takes to have a strong knowledge-oriented culture, though not every aspect of that culture was positive. Chief amongst the issues of importance was communication and the need to overcome communication barriers. Respondents cited many communication barriers between management and workers, and between the DAC's training and engineering directorates. These barriers inhibited the flow of senior leader values and expectations, stories of success and failure, and policy or procedural changes. To combat these barriers, the DAC regularly circulates two newsletters aimed at publicizing accomplishments and best practices as well as management staff meeting notes.

The DAC also demonstrates a deep appreciation for education, especially for professional and degree seeking opportunities. Such emphasis was noted by way of senior leader encouragement, availability of educational programs, and extensive interpersonal networks of personnel engaged in their own programs of study. Respondents also reported cultural support for knowledge flow; no one felt that existing policies and procedures necessarily inhibited attempts to share knowledge.

Although culture per se was not perceived to hinder to innovation, respondents were somewhat ambivalent in that innovation was also not highly encouraged. The same cannot be said of the incentive structure. Due to the funding processes within the DAC, different directorates must "sell" their programs in order to receive their requested share of the annual budget - this incentive for competition has increasingly stifled knowledge flow between directorates, especially while budgetary decisions were still pending. On a more personal level, no explicit incentives or reward program had been instituted for knowledge-sharing or knowledge work that led to innovation or other positive organizational outcomes. In fact, the only incentives available for recognizing excellence or superior contribution to the DAC's knowledge processes was verbiage and language indicating as such in an employee's annual performance evaluation.

\subsection{KMS Fit model analysis and construction}

Respondents were next asked to describe their perceptions and experiences of how each construct in the research model was related to the others in accordance with the construct pairings described in the Section 3 (not all possible combinations were analyzed); aggregate responses appear in Table 1. Highlighted rows indicate conflicts of perception where a majority of respondents perceived a relationship between constructs but were evenly split as to the direction of influence. These conflicts are accounted for during model rationalization. Additional pairings are denoted with an asterisk. Here, one data point was eliminated based upon obvious interpretation errors. Specifically, definitions 
were provided for each construct of interest. Respondents could reference the definitions at any time; however, one participant's description of the knowledge task in relation to other constructs was clearly articulated in terms of performing the knowledge task rather than the nature of the task itself. Consequently, the "vote" cast by that individual for those particular pairings was removed; this is a limitation of the current study.

Table 1: Aggregate Construct Interrelationship Data

\begin{tabular}{|c|c|c|c|c|}
\hline Construct "A" & Construct "B" & $\leftarrow$ & $\longrightarrow$ & No Relationship \\
\hline Cognitive Barriers & KMS Functionality & 2 & 4 & 1 \\
\hline Cognitive Barriers & Intention to Use & 0 & 5 & 2 \\
\hline Cognitive Barriers & Knowledge Markets & 2 & 2 & 3 \\
\hline Cognitive Barriers & Knowledge Networks & 4 & 3 & 0 \\
\hline Cognitive Barriers & Knowledge Task Req. & 1 & $2^{*}$ & 2 \\
\hline Cognitive Barriers & Organizational Culture & 5 & 2 & 0 \\
\hline Cognitive Barriers & Performance & 1 & 6 & 0 \\
\hline Cognitive Barriers & Task/Technology Fit & 3 & 2 & 2 \\
\hline KMS Functionality & Task/Technology Fit & 0 & 7 & 0 \\
\hline Intention to Use & Performance & 3 & 2 & 2 \\
\hline Intention to Use & Task/Technology Fit & 5 & 0 & 2 \\
\hline Knowledge Markets & KMS Functionality & 1 & 4 & 2 \\
\hline Knowledge Markets & Intention to Use & 1 & 5 & 1 \\
\hline Knowledge Markets & Knowledge Networks & 4 & 3 & 0 \\
\hline Knowledge Markets & Knowledge Task Req. & 2 & $1^{*}$ & 1 \\
\hline Knowledge Markets & Organizational Culture & 2 & 2 & 3 \\
\hline Knowledge Markets & Performance & 1 & 6 & 0 \\
\hline Knowledge Markets & Task/Technology Fit & 2 & 3 & 2 \\
\hline Knowledge Networks & KMS Functionality & 2 & 3 & 2 \\
\hline Knowledge Networks & Intention to Use & 1 & 4 & 2 \\
\hline Knowledge Networks & Knowledge Task Req. & 2 & $2^{*}$ & 1 \\
\hline Knowledge Networks & Organizational Culture & 4 & 2 & 1 \\
\hline Knowledge Networks & Performance & 2 & 5 & 0 \\
\hline Knowledge Networks & Task/Technology Fit & 2 & 2 & 3 \\
\hline Knowledge Task Req. & Task/Technology Fit & $1^{*}$ & 3 & 1 \\
\hline Organizational Culture & KMS Functionality & 2 & 4 & 1 \\
\hline Organizational Culture & Intention to Use & 0 & 4 & 3 \\
\hline Organizational Culture & Knowledge Task Req. & $0^{*}$ & 4 & 2 \\
\hline Organizational Culture & Performance & 1 & 6 & 0 \\
\hline Organizational Culture & Task/Technology Fit & 2 & 2 & 3 \\
\hline
\end{tabular}

A matrix was constructed (Table 2) highlighting the directions of influence from Table 2 and sorted in descending order of delta $(\Delta)$. When sorted, the most influential constructs appear at the top of the matrix; these serve as the "drivers" in the model of the perceptual system linking all other constructs together. Organizational Culture (OC) is a "primary driver" because it influences all other constructs, but was not perceived to be influenced by any construct itself. Intention to Use (IU) is a "primary outcome" because it was not perceived to exert influence on any other constructs. The first four constructs "driving" or impacting the rest of the system are all elements of the social ecology (Organizational Culture, Knowledge Networks, Cognitive Barriers, and Knowledge Markets); the remaining five comprise TTF. 
Table 2: Sorted Interrelationship Matrix

\begin{tabular}{||r||c|c|c|c|c|c|c|c|c||c|c|c||}
\hline \hline & CB & F & IU & KM & KN & KT & OC & TTF & P & OUT & IN & $\Delta$ \\
\hline OC & $\uparrow$ & $\uparrow$ & $\uparrow$ & $\uparrow$ & $\uparrow$ & $\uparrow$ & & $\uparrow$ & $\uparrow$ & 8 & 0 & 8 \\
\hline KN & $\uparrow$ & $\uparrow$ & $\uparrow$ & $\uparrow$ & & $\uparrow$ & $\leftarrow$ & $\uparrow$ & $\uparrow$ & 7 & 1 & 6 \\
\hline CB & & $\uparrow$ & $\uparrow$ & $\uparrow$ & $\leftarrow$ & $\uparrow$ & $\leftarrow$ & $\leftarrow$ & $\uparrow$ & 5 & 3 & 2 \\
\hline KM & $\leftarrow$ & $\uparrow$ & $\uparrow$ & & $\leftarrow$ & $\leftarrow$ & $\leftarrow$ & $\uparrow$ & $\uparrow$ & 4 & 4 & 0 \\
\hline KT & $\leftarrow$ & & & $\uparrow$ & $\leftarrow$ & & $\leftarrow$ & $\uparrow$ & & 2 & 3 & -1 \\
\hline F & $\leftarrow$ & & & $\leftarrow$ & $\leftarrow$ & & $\leftarrow$ & $\uparrow$ & & 1 & 4 & -3 \\
\hline TTF & $\uparrow$ & $\leftarrow$ & $\uparrow$ & $\leftarrow$ & $\leftarrow$ & $\leftarrow$ & $\leftarrow$ & & & 2 & 5 & -3 \\
\hline P & $\leftarrow$ & & $\uparrow$ & $\leftarrow$ & $\leftarrow$ & & $\leftarrow$ & & & 1 & 4 & -3 \\
\hline IU & $\leftarrow$ & & & $\leftarrow$ & $\leftarrow$ & & $\leftarrow$ & $\leftarrow$ & $\leftarrow$ & 0 & 6 & -6 \\
\hline \hline
\end{tabular}

In accordance with IQA methodology, a perceptual model was then built to represent the perceived or experienced relationships between constructs based on the data from Table 2. The model was then simplified for parsimony while still retaining the underlying information about the precedence and influence between constructs. For example, Organizational Culture was perceived to impact KMS Functionality; however, Organizational Culture also impacted Knowledge Markets, which in turn impacted KMS Functionality. Organizational Culture thus impacted KMS Functionality through multiple paths (directly and indirectly). The arrow between Organizational Culture and KMS Functionality was removed because the "absolute" connection between constructs is still implied graphically by the indirect relationships between intervening constructs. This process was repeated until no redundant links remained (Figure 3).

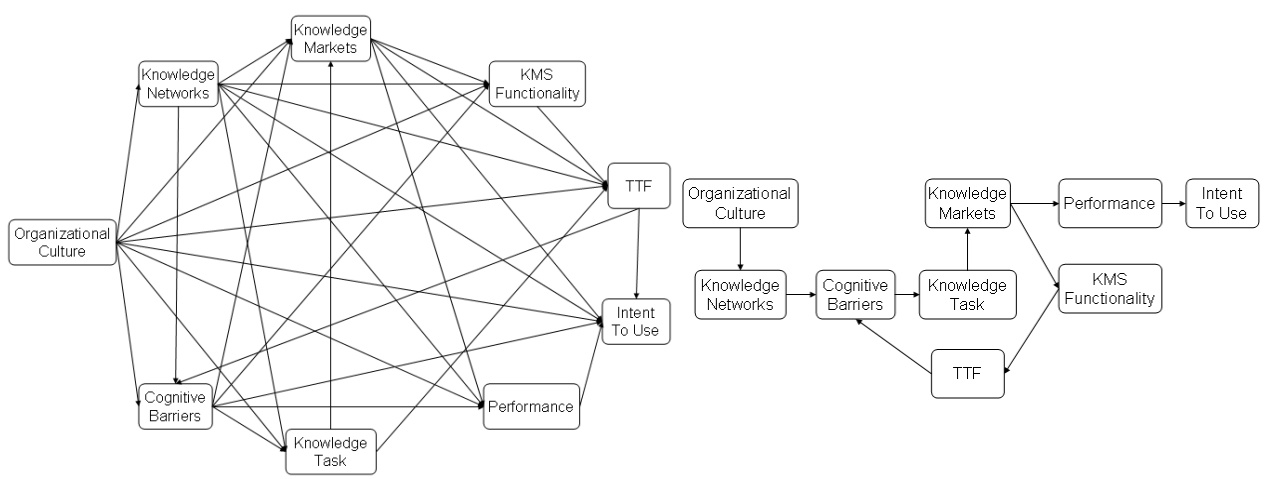

Figure 3. Baseline (left) and Simplified (right) KMS Fit Perceptual Models

Finally, the IQA modeling process accounts for conflicting perceived directions of influence between constructs; adding the conflicts back into the model accounts for this "split" opinion. Only one relationship (highlighted below) had to be added to the model in order to account for all four conflicts. Figure 4 describes how TTF and concepts related to the social ecology "looked" and "interacted" in the minds of the DAC participants. This model provides a unique perspective on the prevalence of the social ecology to the fitness of a KMS for knowledge tasks. 


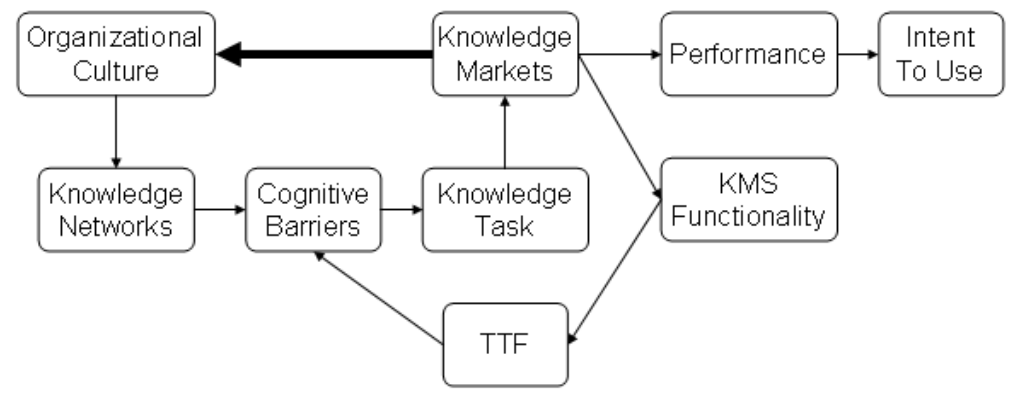

Figure 4. Final Perceptual Model of KMS Fit Constructs

\section{Conclusions and Recommendations}

When comparing the Proposed KMS Fit model to the baseline perceptual model derived from the DAC personnel interviews, all but one (Figure 5; circled in both systems) of the TTF and proposed social ecology-based relationships were supported by the perceptions and experiences of the DAC employees. These observations might suggest that the underlying mechanisms of TTF are not fundamentally different in the context of KM or KMS. However, the topography of the perceptual system and the disparity of the relationship between performance and intentions tell a much more complex story.

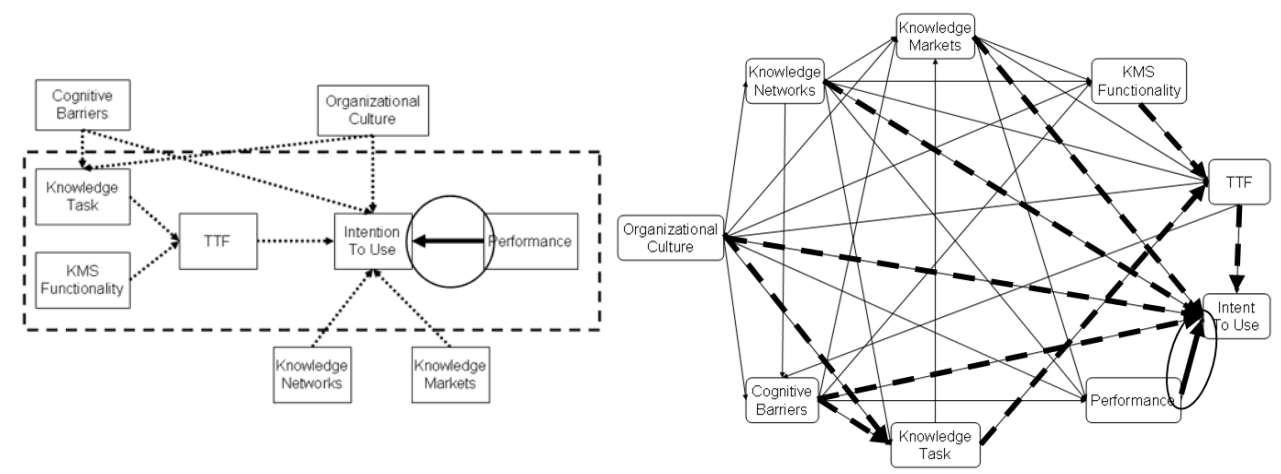

Figure 5. Comparison of KMS Fit Model (left) to Baseline Perceptual Model (right)

\subsection{TTF and the unique context of KM/KMS}

The unanticipated relationship observed between performance and intentions should not be understated. Jennex (2005 \& 2008) observed that the KMS with the greatest impact may in fact not be used all that frequently. The perceptions and experiences of the DAC employees mirror these sentiments - when it comes to a knowledge management system such as the ETS, those same (perhaps infrequent) gains in performance were ultimately what impacted respondents' intentions to return to the ETS to support their knowledge work in the future.

These findings are perhaps unsurprising; it is widely maintained that knowledge and knowledge management in particular should enable some sort of action (Alavi \& Leidner, 1999; Jennex, 2008; Jennex et al., 2007; Nonaka, 1994). For the DAC's knowledge workers, the influence or precedence of performance (action) on intentions was a more 
commonly perceived relationship than that of intentions to performance. Obtained results lend further credence to the notions that KMS may well be a special subset of IS and that the constructs, relationships between constructs, and even dependent variables of interest in the models and frameworks for KMS research, analysis, and assessment (including TTF) should be chosen with care (Jennex, 2005 \& 2008; Jennex \& Olfman, 2004b). Commensurate advice to the champions of a KMS initiative includes performanceoriented training such that the value of the system is demonstrated up front and in context - tied to actual performance-rather than by explicit, but perhaps isolated, demonstrations of system features or functionality.

\subsection{Impact of the social ecology}

The implications of the obtained results are even more telling based on the issues highlighted in Figure 6. In particular, the constructs in the diagram on the left that comprise the social ecology (Organizational Culture, Knowledge Networks, Cognitive Barriers, and Knowledge Markets) are shown to impact every construct internal to the foundational TTF. The prominence and impact of the social ecology factors are translated graphically by the fact that they appear as "drivers" in the system, essentially influencing the value, outcome, or perceptions of all the other constructs. These relationships indicate that perceptions surrounding knowledge and KM-initiatives as related to the task at hand, or to the KMS to be used, are highly dependent upon factors in the social environment, well beyond the foundational issues associated with the task and system characteristics.

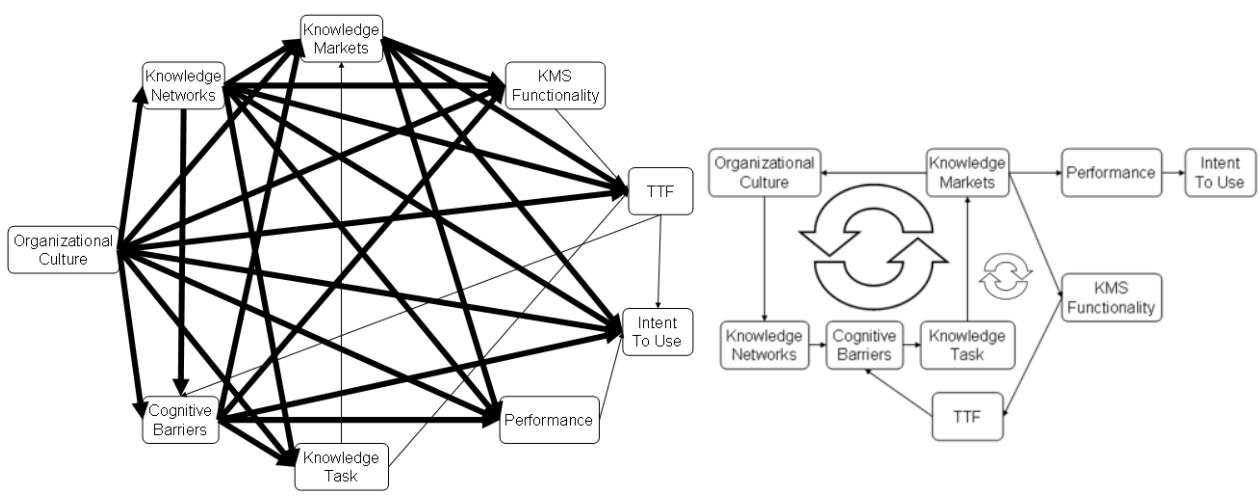

Figure 6. Multiple Influences (left) and Feedback Loops (right) of the Social Ecology

On the right of Figure 6, emphasis has been added to the Perceptual Model of KMS Fit illustrating the "feedback loop" that encompasses the social ecology factors interspersed with the perceptions and experiences of the Knowledge Task Requirements. This feedback loop is centered within another feedback loop encompassing perceptions and experiences of KMS Functionality and TTF. Graphically, these feedback loops indicate near structuration-like patterns of creation, enforcement, and reinforcement of the various factors and mechanisms of influence associated with the underlying TTF model and social ecology constructs. Thus, the system of perception built from the minds and experiences of the DAC employees clearly indicates that the social context, and characteristics of the knowledge environment in which they worked, played a critical role in determining overall KMS Fit - and ultimately it was their intention to return to the KMS, rather than their performance resulting from the use of a KMS, that was perceived as the "outcome" or dependent variable of the system.

\subsection{Limitations and recommendations for future study}


This study represented an initial exploration of the potential adjustments or tailoring that might be warranted to extend the explanatory and predictive power of the TTF framework into KMS-related contexts. Focus was maintained primarily on expanding the scope of TTF rather than attacking its very foundations. As such, only the direct relationships specified in the TTF model were examined via the IQA-based interrelationship analyses while the entire set of possible interrelationships of the social ecology constructs were considered and explored. It is possible that the unique context of knowledge-based work brings to bear additional mechanisms of influence and reinforcement between the TTF-related constructs beyond those few relationships explored in this study. Future such investigations that attempt to build on the complexities of human perception and experience should endeavor to more fully explore all potential avenues of influence between the constructs of interest.

One potential source of error arose from differences in interpretation on the part of the participants - although they were provided a priori construct definitions, they reported their perceptions and experiences based on their own perspectives regarding those constructs. As indicated in Section 4, at least one individual clearly articulated his perceptions and experiences of the knowledge task in terms of performing the task rather than the nature of the task itself. The "word pictures" painted by the participants" descriptions were relatively unaffected by such interpretation errors - construct analysis was based upon the content of the passages in the transcripts. However, the interrelationship table providing the data used to construct the perceptual system of KMS Fit required post-hoc reconciliation. In particular, when the stated direction of influence between constructs (e.g., "I think knowledge markets influence cognitive barriers") were clearly at odds with the definitions or descriptions of those same relationships, the direction of influence entered on the table was drawn from the descriptions and anecdotes in the transcripts as provided by the participants.

Due to logistical constraints, only seven interviews were accomplished. Participants were further limited to a specific subset of DAC employees. As instructors, their outlook on knowledge and knowledge-related issues may well not be shared by all other members of the DAC, much less generalize with any degree of precision to a larger population or other organizations. However, interpretive studies need to be less concerned about statistical generalization per se than they do about the transferability or applicability of the subject matter to similar contexts or similar phenomena (Yin, 2003).

Insomuch as it is reasonable to conclude that the DAC instructors are not entirely dissimilar to other knowledge workers in other large organizations, the observations and implications derived from this study are far from completely suspect. Nevertheless, the IQA methodology, especially in the construction of the system of representation, draws from techniques that translate individual perceptions and experiences into a more collective depiction or understanding of a particular phenomenon. As such, larger groups of respondents are desirable so that no one individual has undue influence on the construction of the system of representation for the entire constituency (Northcutt \& McCoy, 2004).

\subsection{Conclusion}

This study examined a government application of a KMS and sought to determine if Task/Technology Fit mechanisms behaved differently relative to KMS than to traditional information systems. A TTF-based KMS Fit model was developed and evaluated using the lived experiences and perceptions of a small group of government ammunition 
technical training instructors. The social context and characteristics of the environment in which they worked and used the KMS was found to influence key TTF relationships between performance and intentions, and played a critical role in determining overall KMS Fit. Given the large amount of money spent on KMS annually, the model developed in this study may serve as a starting-point for future research aiding KMS development and ensuring success of KMS investments.

\section{References}

1 Alavi, M., \& Leidner, D. E. (1999). Knowledge management systems: Issues, challenges, and benefits. Communications of the Association for Information Systems, 1(2es), 1-37.

2 Alavi, M., \& Leidner, D. E. (2001). Knowledge management and knowledge management systems: Conceptual foundations and research issues. MIS Quarterly, 25(1), 107-136.

3 Argote, L., \& Ingram, P. (2000). Knowledge transfer: A basis for competitive advantage in firms. Organizational Behavior and Human Decision Processes, 82(1), 22.

4 Baloh, P. (2007). The role of fit in knowledge management systems: Tentative propositions of the KMS design. Journal of Organizational and End User Computing, 19(4), 22-41.

5 Brown, J. S., \& Duguid, P. (1991). Organizational learning and communities-ofpractice: Toward a unified view of working, learning, and innovation. Organization Science, 2(1), 40-57.

6 Chua, A., \& Lam, W. (2005). Why KM projects fail: A multi-case analysis. Journal of Knowledge Management, 9(3), 6-17.

7 Davenport, T. H., \& Prusak, L. (2000). Working knowledge: How organizations manage what they know. Boston: Harvard Business School Press.

8 Davis, F. D., Bagozzi, R. P., \& Warshaw, P. R. (1989). User acceptance of computer technology: A comparison of two theoretical models. Management Science, 35(8), 982-1003.

9 DeLone, W. H., \& McLean, E. R. (1992). Information systems success: The quest for the dependent variable. Information System Research, 3(1), 60-95.

10 DeLone, W. H., \& McLean, E. R. (2002). Information systems success revisited. Paper presented at the 35th Hawaii International Conference on System Sciences, Hawaii.

11 Dishaw, M. T., \& Strong, D. M. (1999). Extending the technology acceptance model with task-technology fit constructs. Information \& Management, 36, 9-21.

12 Goodhue, D. L. (1998). Development and measurement validity of a task-technology fit instrument for user evaluations of information systems. Decision Sciences, 29(1), 105-138.

13 Goodhue, D. L., \& Thompson, R. L. (1995). Task-technology fit and individual performance. MIS Quarterly, 19(2), 213-236.

14 Grover, V., \& Davenport, T. H. (2001). General perspectives on knowledge management: Fostering a research agenda. Journal of Management Information Systems, 18(1), 5-21. 
15 Gupta, A. K., \& Govindarajan, V. (2000). Knowledge management's social dimension: Lessons from Nucor Steel. MIT Sloan Management Review, 42(1), 71-80.

16 Huber, G. P. (2001). Transfer of knowledge in knowledge management systems: Unexplored issues and suggested studies. European Journal of Information Systems, $10,72-79$.

17 INPUT (2005). INPUT predicts federal knowledge management spending will reach $\$ 1.3$ billion by FY10. Retrieved December 3, 2008 from http://www.input.com/corp/press/detail.cfm?news=1091

18 Jarvenpaa, S.L., and Majchrzak, A. (2005). Developing individuals' transactive memories of their ego-centric networks to mitigate risks of knowledge-sharing: The case of professionals protecting cyberSecurity. Proceedings of the International Conference on Information Systems, 2005.

19 Jennex, M. E. (2008). Exploring system use as a measure of knowledge management success. Journal of Organizational and End User Computing, 20(1), 50-63.

20 Jennex, M. E., \& Olfman, L. (2004a). Assessing knowledge management success/effectiveness models. Paper presented at the 37th Hawaii International Conference on System Sciences, Hawaii.

21 Jennex, M. E., \& Olfman, L. (2004b). Modeling knowledge management success. Paper presented at the Conference on Information Science and Technology Management.

22 Jennex, M. E., Smolnik, S., \& Croasdell, D. (2007). Towards defining knowledge management success. Paper presented at the 40th Annual Hawaii International Conference on System Sciences, Hawaii.

23 Mathieson, K., \& Keil, M. (1998). Beyond the interface: Ease of use and task/technology fit. Information \& Management, 34, 221-230.

24 McGreevy, Maura (2007). AMR Research finds spending on knowledge management will hit $\$ 73 \mathrm{~B}$ in 2007. Retrieved December 3, 2008, from www.amrresearch.com/Content/View.asp?pmillid=20768

25 Nonaka, I. (1994). A dynamic theory of organizational knowledge creation. Organization Science, 5(1), 14-37.

26 Northcutt, N., \& McCoy, D. (2004). Interactive qualitative analysis: A systems method for qualitative research. Thousand Oaks, CA: Sage.

27 Osterloh, M., \& Frey, B. S. (2000). Motivation, knowledge transfer, and organizational forms. Organization Science, 11(5), 538-550.

28 Polanyi, M. (1962). Tacit knowing: Its bearing on some problems of philosophy. Reviews of Modern Physics, 34(4), 601-616.

29 Seddon, P. B., \& Kiew, M.-Y. (1996). A partial test and development of DeLone and McLean's model of IS success. Australian Journal of Information Systems, 4(1), 90108.

30 Storey, J., \& Barnett, E. (2000). Knowledge management initiatives: Learning from failure. Journal of Knowledge Management 4(2), 145-156.

31 von Krogh, G. (1998). Care in knowledge creation. California Management Review, 40(3), 133-153.

32 Wiig, K. M. (1997). Knowledge management: Where did it come from and where will it go? Expert Systems with Applications, 13(1), 1-14.

33 Yin, R. K. (2003). Case study research: Design and methods. Thousand Oaks, CA: Sage Publications, Inc. 